ARTICLE

Received 4 Oct 2012 | Accepted 5 Jun 2013 | Published 5 Jul 2013

DOI: $10.1038 /$ ncomms3115

\title{
R-loops and nicks initiate DNA breakage and genome instability in non-growing Escherichia coli
}

\author{
Hallie Wimberly ${ }^{1,}$, Chandan Shee $^{1}$, P. C. Thornton ${ }^{1}$, Priya Sivaramakrishnan ${ }^{1}$, Susan M. Rosenberg ${ }^{1,2}$ \\ \& P. J. Hastings ${ }^{1}$
}

Double-stranded DNA ends, often from replication, drive genomic instability, yet their origin in non-replicating cells is unknown. Here we show that transcriptional RNA/DNA hybrids (R-loops) generate DNA ends that underlie stress-induced mutation and amplification. Depleting RNA/DNA hybrids with overproduced RNase $\mathrm{HI}$ reduces both genomic changes, indicating RNA/DNA hybrids as intermediates in both. An Mfd requirement and inhibition by translation implicate transcriptional R-loops. R-loops promote instability by generating DNA ends, shown by their dispensability when ends are provided by I-Scel endonuclease. Both $\mathrm{R}$-loops and single-stranded endonuclease Tral are required for end formation, visualized as foci of a fluorescent end-binding protein. The data suggest that R-loops prime replication forks that collapse at single-stranded nicks, producing ends that instigate genomic instability. The results illuminate how DNA ends form in non-replicating cells, identify R-loops as the earliest known mutation/amplification intermediate, and suggest that genomic instability during stress could be targeted to transcribed regions, accelerating adaptation.

\footnotetext{
${ }^{1}$ Department of Molecular and Human Genetics, 1 Baylor Plaza, Houston, Texas 77030, USA. ${ }^{2}$ Departments of Biochemistry and Molecular Biology, Molecular Virology and Microbiology and the Dan L Duncan Cancer Center, Baylor College of Medicine, Houston, Texas 77030, USA. †Present address: Department of Pathology, Yale University School of Medicine, 333 Cedar Street, New Haven, Connecticut 06510, USA. Correspondence and requests for materials should be addressed to P.J.H. (email: hastings@bcm.edu).
} 
$\mathrm{D}$ NA double-strand breaks and ends (DSBs/DSEs) are the single most potent inducers of genomic instability in cells from bacteria to human. Imperfectly accurate repair of DSBs/DSEs causes genomic rearrangements and small mutations that underlie breast ${ }^{1}$ and other cancers ${ }^{2}$, many human genetic diseases ${ }^{3}$, evolution of pathogenic bacterial biofilms ${ }^{4}$, mutations that cause bacterial antibiotic resistance ${ }^{5}$, bacterial evasion of host defenses ${ }^{6}$, and stress-induced mutations (SIMs) that may accelerate evolution preferentially when cells are maladapted to their environments ${ }^{7-9}$. Although DNA breaks result from exogenous DNA-damaging agents, endogenous or spontaneous DSBs/DSEs are thought to be the commonest instigators of genomic instability mechanisms in all cells (for example, Vilenchik and Knudson ${ }^{10}$ ). However, despite their paramount importance to genomic instability that drives evolution and disease, and although their rates of formation are being quantified $^{11}$, mechanisms of spontaneous DNA breakage are poorly understood.

Some mechanisms that produce spontaneous DSBs/DSEs are associated with DNA replication. First, when replication forks encounter single-stranded (ssDNA) breaks in the DNA template, they can 'collapse' producing a single DSE ${ }^{12}$ (illustrated Fig. 1e,f). Second, paused replication forks can be cleaved by endonucleases that cleave recombination intermediates creating DSEs ${ }^{13,14}$. Third, when converging replication and transcription complexes collide 'head on' on DNA, DSBs/DSEs can result ${ }^{15,16}$, though the precise mechanism of breakage is not known ${ }^{17}$. Fourth, DSBs/ DSEs also result from co-directional collisions of the replisome with RNA polymerase (RNAP), when RNAP has 'back-tracked' on the DNA template ${ }^{17,18}$. DSEs produced by co-directional collisions are prevented by Mfd helicase, which dislodges the back-tracked RNAP 18 . Formation of these DSEs requires an RNA/DNA hybrid ${ }^{18}$ and appears to result from the DNA polymerase stopping at the RNA/DNA hybrid 'under' the backtracked RNAP. This leaves a ssDNA nick or gap in the new DNA strand ${ }^{18,19}$, which then becomes a DSE by fork collapse when a second round of replication encounters the nick (per Fig. 1e,f). Thus, DSEs generated by co-directional collisions of the replisome with RNAP are: (1) prevented by Mfd helicase, and (2) caused by RNA/DNA hybrids plus replication.

Whereas the mechanisms of DSB/DSE generation listed above require replicating DNA, DSBs/DSEs also provoke SIM pathways in starvation-stressed cells that are unlikely to be replicating DNA. DSBs/DSEs initiate two mechanisms of SIM in starving Escherichia coli cells: stress-induced point mutation ${ }^{9,20,21}$ and gene amplification ${ }^{21,22}$ (reviewed in refs 7-9). Both mechanisms occur via repair of DSBs/DSEs that becomes mutagenic upon activation of the RpoS-controlled general stress respons ${ }^{23}$. The point mutation mechanism is an RpoS-controlled switch to mutagenic DSE repair in which replication primed from DSEs uses error-prone DNA polymerases when RpoS is activated, causing indel and base-substitution mutations ${ }^{9,21,24,25}$ (illustrated Fig. 1g,k). The amplification mechanism causes genome rearrangements $22,26,27$ (reviewed in Hastings et al. ${ }^{28}$ ) is also thought to result from DSE-initiated replication ${ }^{21,22}$ (Fig. $1 \mathrm{~g}-\mathrm{j}$ ) and requires $\mathrm{RpoS}^{23}$. Thus, parts g-k of Fig. 1 represent current

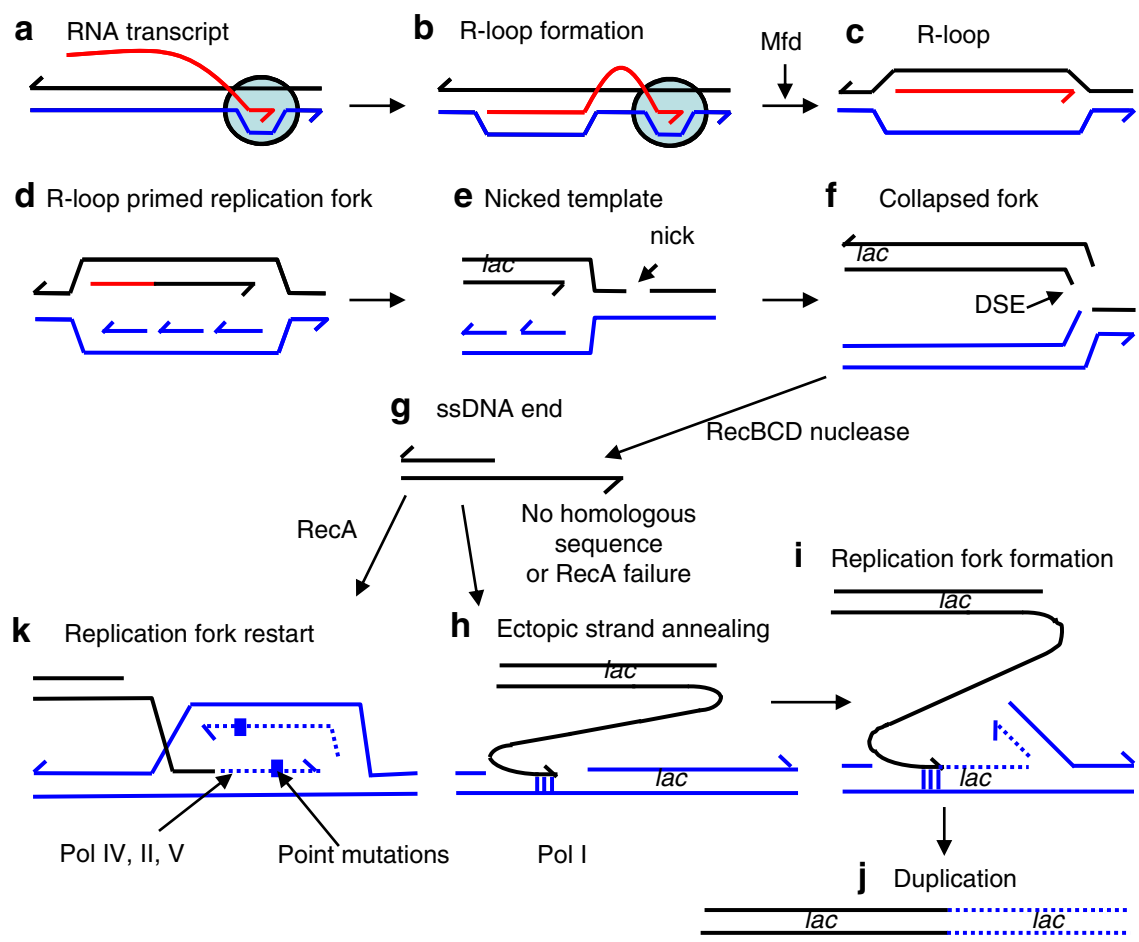

Figure 1 | Model for transcription-promoted R-loop initiation of DSEs. (a-c) R-loops form by incorporation of the transcript (red) into supercoiled DNA (blue/black) behind the site of transcription ${ }^{41}$. R-loop formation is usually inhibited by ribosomes or the R-loop removed by RNase HI. (b) Stalled RNAP (circle) (c) is removed by $\mathrm{Mfd}^{38}$. (d) The R-loop can then form a replication fork ${ }^{35}$. (e) If the replication fork encounters a nick in a template DNA strand, the fork will collapse (f), forming a single DSE ${ }^{12}$. In stressed cells, the collapsed fork might be repaired and restarted by microhomology-mediated break-induced replication $(\mathbf{g}-\mathbf{j})^{26}$ producing genome rearrangements (duplication shown here). (g) DSE degradation and $5^{\prime}$-end resection by RecBCD might be followed by $\mathbf{h}$ annealing of the overhanging $3^{\prime}$-end to sSDNA at a site of microhomology (vertical lines), shown here in the lagging-strand template of another replication fork (blue). (i,j) This replication restart is shown at a position behind where the initial fork collapsed, so that a segment of the genome including the lac region becomes duplicated. The duplication can be expanded into an amplified array by unequal crossing-over (not illustrated). (k) Alternatively, point mutation is proposed to occur when the DSE is repaired by homologous recombination-mediated replication-fork restart using error-prone polymerase Pol IV during restart due to licensing of Pol IV (and also Pols II and V) by the RpoS and SOS stress responses ${ }^{9,21,24}$. 
understanding based on much previous work. Stress-induced point mutation occurs both in a Lac plasmid-based mutation assay $^{29}$ and in chromosomes of plasmid-free cells ${ }^{9,24,25,30}$, and underlies half of spontaneous base-substitution and frameshift mutagenesis in the chromosomes of starving E. coli ${ }^{24}$, and so is likely to be important to evolution. Amplification is an important model for mechanisms of human copy-number variation ${ }^{28}$.

The origin of DSBs/DSEs in non-replicating cells, including the DSEs that that underlie SIM, is poorly understood. At sites in an $\mathrm{F}^{\prime}$ plasmid, SIM requires TraI ssDNA endonuclease ${ }^{21}$, which makes ssDNA nicks in the F plasmid. As TraI is not needed when DSBs are delivered to the F using double-stranded endonuclease I-SceI, ssDNA nicks are inferred to promote SIM by causing DSB/ $\mathrm{DSEs}^{21}$, for example via fork collapse (Fig. 1e,f). Cells that express a phage ssDNA endonuclease and carry its cleavage site in the $\mathrm{F}^{\prime}$ do not require TraI for point mutation, implying that any ssDNA nick will allow SIM $^{31}$. However, spontaneous DSBs/DSEs also promote mutation in starving plasmid-free $E$. coli $i^{9,24}$, indicating that DSBs/DSEs also form independently of Tral. Given that replication is repressed during starvation, that at least some of the SIM-promoting DSEs probably arise from fork collapses ${ }^{21,31,32}$, and that most models for the origins of spontaneous DSEs involve replication, how spontaneous DSEs form in starvation-stressed non-growing cells is unclear. If fork collapse is the mechanism, then how and where do the forks originate? If not, then how are the ssDNA nicks converted into the DSEs that underlie SIM? How spontaneous DSEs are formed in non-replicating cells, and how replication-dependent processes (for example, Fig. 1) can occur in starved, non-replicating cells are important and fundamental problems, the solution to which is likely to pertain to many different organisms and circumstances.

DNA replication in growing cells is initiated at fixed sites (origins). Origin activity is repressed in stationary-phase cells ${ }^{33}$.
However, origin-independent replication can be initiated by RNA paired with unbroken DNA (R-loops, Fig. 1c,d) and at D-loops (similar structures made solely of DNA) under some circumstances $^{34,35}$. Here, we provide evidence that DSE production is promoted by R-loops in starving cells.

We show that RNA/DNA hybrids produced by transcription (R-loops) are precursors to the DSBs/DSEs that initiate stressinduced amplification (genome rearrangement) and point mutation, making R-loops the earliest known molecular intermediate for both processes. We show that R-loops provoke DSBs/DSEs by a two-step mechanism that is unlike the co-directional collision mechanism. We suggest that R-loop-primed replication forks collapse at independent ssDNA nicks to generate DSEs (Fig. 1a-f). This mechanism may explain DSE formation in many circumstances, particularly in non-replicating cells in which firing of standard replication origins is suppressed.

\section{Results}

RNA/DNA hybrids underlie stress-induced genomic changes. We used the E. coli Lac assay for stress-induced point mutation ${ }^{29}$ and gene amplification ${ }^{36}$. In this assay, cells carrying a lac $+1 \mathrm{bp}$ frameshift allele in an $\mathrm{F}^{\prime}$ plasmid are grown to saturation in liquid, then spread on solid lactose minimal medium on which they starve. $\mathrm{Lac}^{+}$revertant colonies are of three types (for example, Fig. 2a): generation-dependent mutants that occurred during the liquid growth of the culture before starvation on lactose appear as colonies on day 2 (ref. 29); and stress-induced point-mutant and lac-amplified clones, formation of which requires activation of the RpoS stress response ${ }^{23}$, are visible as $\mathrm{Lac}^{+}$colonies from day 3 onward. Point mutants dominate early (for example, Fig. 2a), and amplified clones rise from $\sim 5 \%$ of $\mathrm{Lac}^{+}$colonies on day 5 to $\sim 40 \%$ by day 8 (ref. 36). Point-mutant
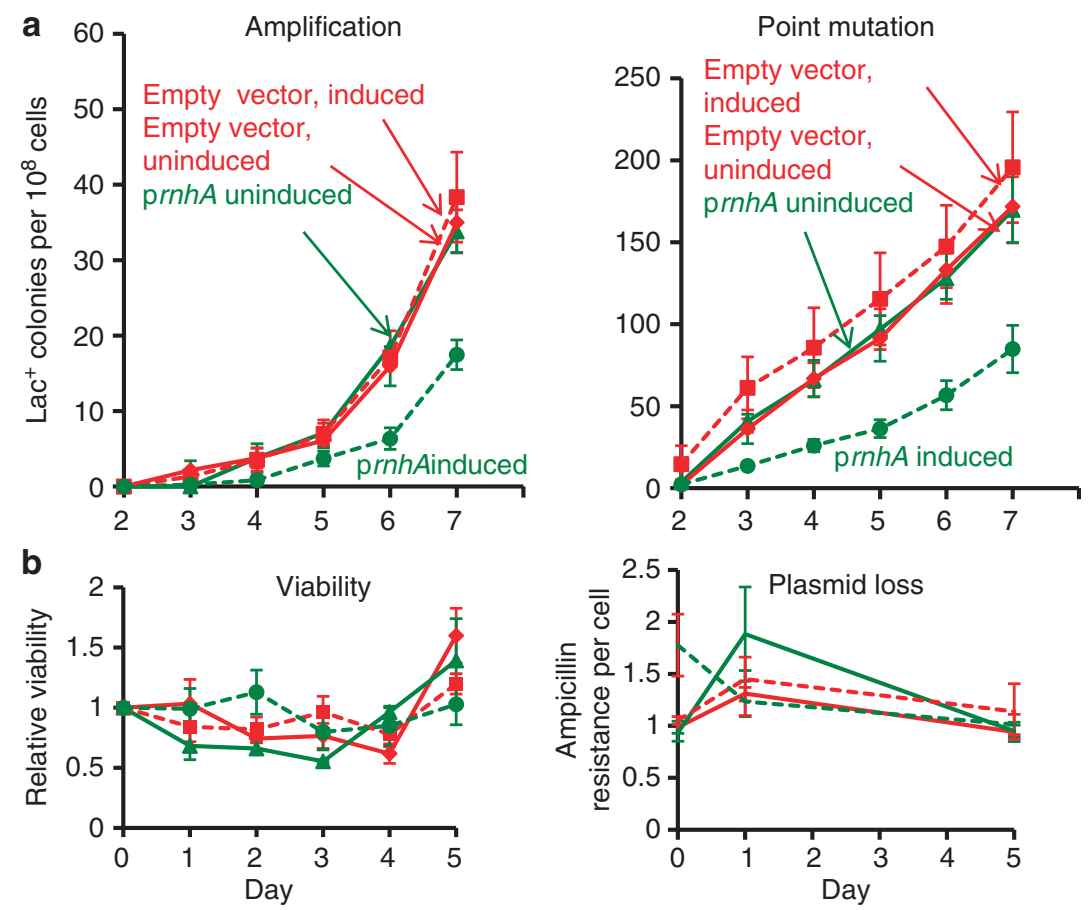

Figure 2 | Overproduction of RNase HI reduces stress-induced amplification and point mutation. (a) Gene amplification and point mutation in strain PJH1093, carrying prnhA (pBAD18-rnhA). Production of RNase $\mathrm{HI}$ is uninduced $(\boldsymbol{\Lambda})$ (solid line) or induced ( $\boldsymbol{\bullet}$ ) (broken line) by the presence of arabinose. Also strain PJH1091, carries empty vector pBAD18 as a control, with transcription from the $\mathrm{P}_{B A D}$ promoter induced ( ) or uninduced ( $\mathbf{a}$ ) by presence or absence of arabinose respectively. (b) Viability and retention of pBAD18-rnhA by day 5 of the experiment shows no plasmid loss. Viability and plasmid loss plots are offset by 2 days: the time needed to form a visible Lac ${ }^{+}$colony. Error bars, one s.e.m. of four parallel cultures. These and all experiments were performed three times with comparable results. 
and amplified colonies are distinguished by appearance on colorindicator medium (Methods).

We find that overproduction of RNase HI, which degrades the RNA from RNA/DNA hybrids ${ }^{37}$, reduces stress-induced amplification and point mutation (Fig. 2a). Preliminary experiments showed that overproduction of RNase HI, encoded by $r n h A$, from the arabinose-inducible $\mathrm{P}_{B A D}$ promoter in plasmid pBAD18-rnhA, inhibits growth. We used the highest concentration of arabinose that did not inhibit growth of cells containing this plasmid $\left(5 \times 10^{-8}(\mathrm{w} / \mathrm{v})\right)$. Overproduction of RNase HI-reduced amplification and point mutation to about $60 \%$ (representative experiments, Fig. 2a), presumably by reducing levels of RNA/DNA hybrids. In three experiments, amplification was reduced to $56 \pm 8 \%$, days $2-7 \quad(P=0.017$, Student's $t$-test). For point mutation, the decrease was to $57 \pm 14 \%$, days 2 to $7,(P=0.047$, Student's $t$-test $)$. There was no loss of pBAD18-rnhA or loss of cell viability during the experiment (Fig. 2b). Amplification and point mutation rates were unaffected by the empty vector with or without arabinose, and were the same as in cells uninduced for pBAD18-rnhA (Fig. 2a). These data imply that about half of amplification and point mutagenesis requires an RNA/DNA hybrid intermediate, and that removal of the RNA/DNA hybrid by overproduced RNase HI curtails both processes.

Loss of RNase HI increases amplification and mutation. We find that knock out of RNase HI increases both amplification and point mutation $\sim 3$-fold (Fig. 3 ). In three experiments, amplification was increased by $2.9 \pm 0.01$-fold, days $2-7 \quad(P=0.003$, Student's $t$-test), and point mutation by $3.1 \pm 0.3$-fold, days $2-5$, $(P=0.006$, Student's $t$-test). Thus, failure to remove RNA/DNA hybrids promotes both processes. Cell viability was unaffected. These data support the conclusion that RNA/DNA hybrids promote both amplification and point mutation.

Instability promoted by RNA/DNA hybrids requires Mfd. Mfd helicase is a transcription accessory protein that associates with $\mathrm{RNAP}^{38}$, couples nucleotide-excision repair to transcription in transcription-coupled repair $^{39}$, and, when a replication folk collides with active RNAP, mediates the conflict by removing $\mathrm{RNAP}^{40}$. Mfd prevents the formation of DSBs/DSEs caused by co-directional collisions of the replisome with $\mathrm{RNAP}^{18}$. By removing stalled RNAP, Mfd also frees the $3^{\prime}$-terminus of the nascent transcript. Processes affected by Mfd are inferred to involve transcription.

We find that the increased amplification and point mutation seen in RNase HI-defective strains requires Mfd (representative experiment, Fig. 3a,b). This indicates that the RNA/DNA hybrids that promote amplification and mutation arise from transcription, and are in the form of R-loops. Knock out of Mfd also decreases amplification in RNase HI-proficient cells to about half (Fig. 3a). In three experiments, amplification was reduced to $0.63 \pm 0.08$, days $2-7(P=0.023$, Student's $t$-test $)$. For point mutation, the decrease was less, but significant $(0.75 \pm 0.02$-fold, days $2-7, P=0.002$, Student's $t$-test). This result resembles the two-fold reduction of amplification and point mutation by overproduction of RNase HI (Fig. 2a), implying that Mfd also promotes those R-loop-instigated genomic changes. These data support the conclusion that at least half of mutagenesis and amplification in wild-type cells proceeds through RNA/DNA hybrid intermediates and that these intermediates are R-loops at sites of transcription.

Translation inhibition increases instability. Formation of transcriptional R-loops in bacteria is inhibited by ribosomes on the nascent transcript ${ }^{41}$ such that inhibiting protein synthesis increases levels of transcription-generated R-loops. We find that pulse-inhibition of ribosome progression increases amplification and point mutation (Fig. 4), supporting the hypothesis that both are promoted by transcriptional R-loops. We treated stationaryphase cultures with spectinomycin, an inhibitor of ribosome translocation $^{42}$, for $3 \mathrm{~h}$ before plating on lactose medium and observed a $\sim 2$-fold increase in amplification (2.2 \pm 0.4 -fold, three experiments, $P=0.048$, Student's $t$-test). Point mutation increased less but still significantly $(1.23 \pm 0.06$-fold, three experiments, $P=0.024$, Student's $t$-test) (Fig. 4a,b). These increases require Mfd (Fig. 4a,b), supporting the transcriptional origin of the spectinomycin-induced increases. The increased point mutation caused by translational inhibition (Fig. 4c) and loss of RNase HI (Fig. 3c) both require DinB/Pol IV, indicating that it occurs by the usual Pol IV-dependent ${ }^{43}$ pathway.

Supporting the hypothesis that spectinomycin increases mutation by increasing R-loop formation, we find that overproduction of RNase HI suppresses spectinomycin-enhanced
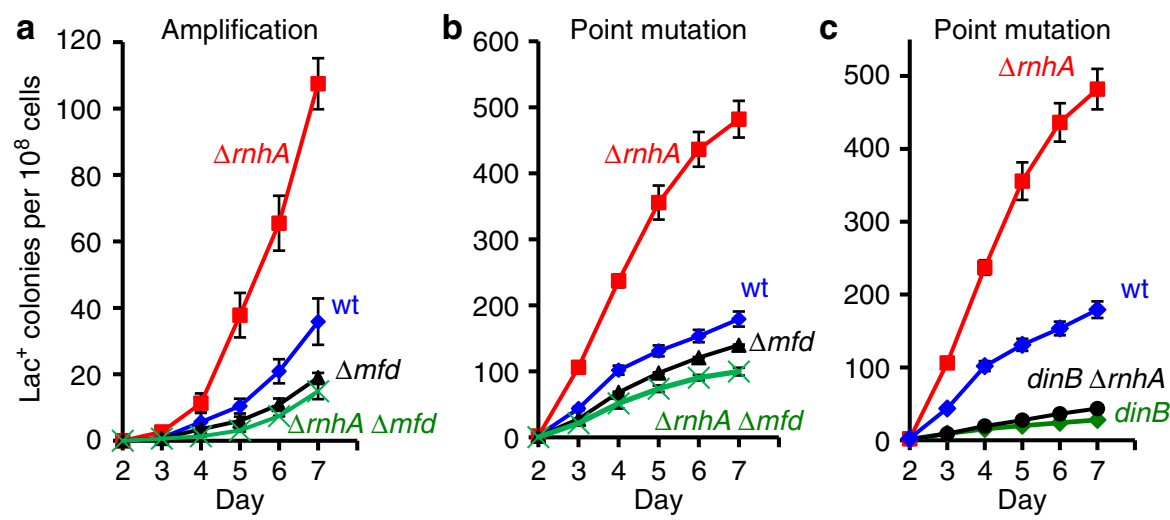

Figure 3 | Loss of RNase $\mathbf{H I}$ increases amplification and point mutation. (a,b) Mfd is required for approximately half of amplification and point mutation in $r n h A^{+}$('wild-type') cells and all the increase in point mutation and amplification in RNase HI-defective $(\Delta$ rnhA) cells. Gene amplification and point mutation in (a) and (b); wild-type $(\bullet), \Delta r n h A(\boldsymbol{\square}), m f d(\boldsymbol{\Delta})$, and $m f d \Delta r n h A(\times)$ strains SMR4562, PJH683, PJH813 and PJH946. (c) Increased point mutation in cells lacking RNase HI requires DinB/DNA Pol IV, as in RNase HI-proficient cells ${ }^{43}$. Wild-type $(\bullet), \Delta r n h A(\boldsymbol{\square}), \operatorname{dinB} 10(\bullet), \operatorname{dinB10} \Delta r n h A$ (-) strains SMR4562, PJH683, SMR5830 and PJH791. Error bars, one s.e.m. of four parallel cultures. These experiments were performed three times with comparable results. 

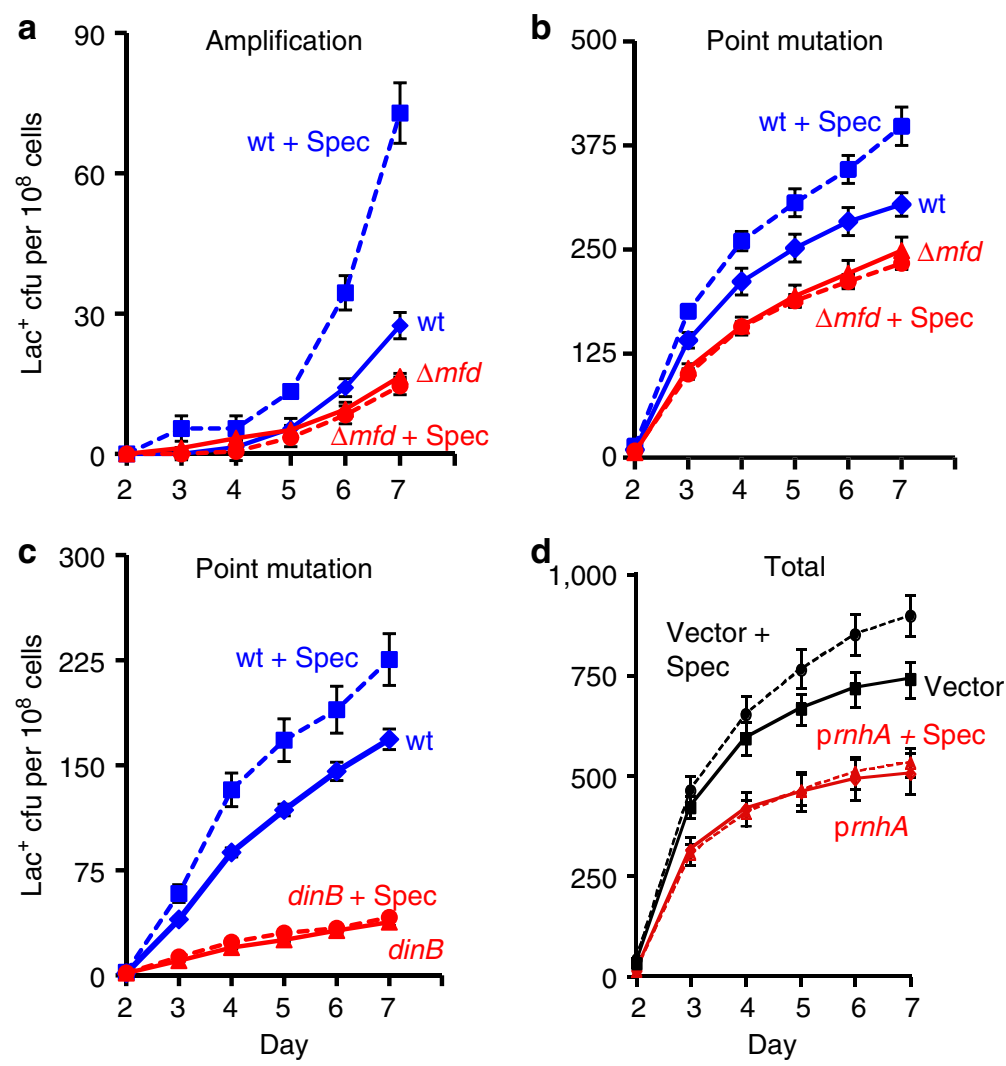

Figure 4 | Inhibition of translation increases amplification and point mutation. Gene amplification (a) and point mutation (b) in WT (SMR4562) and mfd (PJH813) strains pulsed with spectinomycin (spec) (broken lines). WT $+\operatorname{spec}(\boldsymbol{\square})$, WT ( ), mfd $+\operatorname{spec}(\boldsymbol{O})$, mfd ( $(\boldsymbol{\Delta})$. (c) The spectinomycin treatmentinduced increase in point mutagenesis is DinB/Pol IV-dependent. Point mutation in WT (SMR4562) and dinB (SMR5830) cells pulsed with spec.

WT $+\operatorname{spec}(\boldsymbol{\square}), \mathrm{WT}(\bullet), \operatorname{din} B+\operatorname{spec}(\boldsymbol{\bullet}), \operatorname{din} B(\boldsymbol{\Delta})$. The curves for the spectinomycin-treated WT cells differ from those of the untreated WT cells significantly, WT and spec-treated WT differ (for three experiments, $P=0.002$ for point mutation and 0.001 for amplification, Student's $t$-test); $m f d$ and spec-treated $\mathrm{mfd}$ are not significantly different ( $P=0.6$ for point mutation, and 0.7 for amplification). (d) Spectinomycin treatment does not increase $\mathrm{Lac}^{+}$revertants in cells overproducing RNase HI. Vector pBAD18 ( $\left.\mathbf{\square}\right)$, vector pBAD18 spec-treated ( $)$, pBAD18-rnhA ( $)$, pBAD18-rnhA spec-treated $(\boldsymbol{\Lambda})$; broken lines denote spectinomycin treatment. Error bars represent one s.e.m. of four parallel cultures. These experiments were performed three times with comparable results.

mutagenesis (Fig. 4d). Spectinomycin increased mutation in the control-plasmid-carrying strain $1.34 \pm 0.09$-fold (mean \pm s.e.m. of four experiments) but not the RNase HI-overproducing strain (Fig. 4d), which, with or without spectinomycin treatment, showed $0.58 \pm 0.23$ of the level of mutation in the untreated control-plasmid strain (Fig. 4d, mean \pm s.e.m. of four experiments). We conclude that the mutation-promoting effect of spectinomycin is prevented by removal of RNA/DNA hybrids. Although inhibition of translation might affect cells in various ways, both the Mfd-dependence (Fig. 4a,b) and the dependence on RNA/DNA hybrids (Fig. 4d) support the interpretation that translation inhibition promotes amplification and mutagenesis by stabilizing transcriptional R-loops.

Endonuclease-induced breaks substitute for R-loops. In principle, R-loops might promote SIM by promoting DSB/DSEs (Fig. 1b-f) or by promoting some stage of the SIM mechanisms downstream of DSE formation (Fig. 1g-k). Stress-induced point mutation and amplification mechanisms can be separated experimentally into the stages that occur before or after formation of DSEs (Fig. 1g-k, reviewed in Rosenberg et al. ${ }^{9}$ and Hastings et $\left.a l^{28}\right)$. Proteins or DNA intermediates that promote SIM because they promote DSE formation are not required if DSBs are created near lac with I-SceI site-specific endonuclease ${ }^{21}$, as shown for TraI ssDNA endonuclease $e^{21}$ and the major role of the $\sigma^{\mathrm{E}}$ response $^{44}$. By contrast, proteins that promote SIM at stages after creation of DSEs remain required when DSBs are provided by I-SceI (near $l a c^{21}$, or near chromosomal mutation-reporter genes $^{24,25,30}$ ). We used I-SceI to address whether R-loops promote SIM by acting before or after DSE creation. Although I-SceI decreases viability, the RNase HI plasmid does not (Fig. 5c), allowing us to compare the rates of Lac reversion with and without the RNase HI plasmid.

Figure $5 \mathrm{~b}$ shows that when DSBs are made by I-SceI, $\mathrm{Lac}^{+}$ reversion is unaffected by overproduction of RNase HI. This contrasts with reduction of Lac reversion caused by RNase HI overproduction in cells without I-SceI-induced DSBs, either when no I-SceI is present (Fig. 2a) or in the presence of the I-SceI enzyme but no cutsite (Fig. 5a). We conclude that R-loops become superfluous for mutagenesis when a DSB is provided independently.

In three experiments, in the absence of I-SceI cutting ('enzymeonly' control), RNase $\mathrm{HI}$ overproduction reduced the $\mathrm{Lac}^{+}$ reversion rate by a significant $1.7 \pm 0.2$-fold (mean \pm s.e.m.) relative to the control plasmid ( $\mathrm{Lac}^{+}$colonies per day, day 3 to day 5). In contrast, when DSBs were induced, RNase HI overproduction caused $1.23 \pm 0.06$ times more $\mathrm{Lac}^{+}$reversion than seen with the control plasmid. The lack of effect of RNase $\mathrm{H}$ overproduction in strains with I-SceI cuts is significantly different 

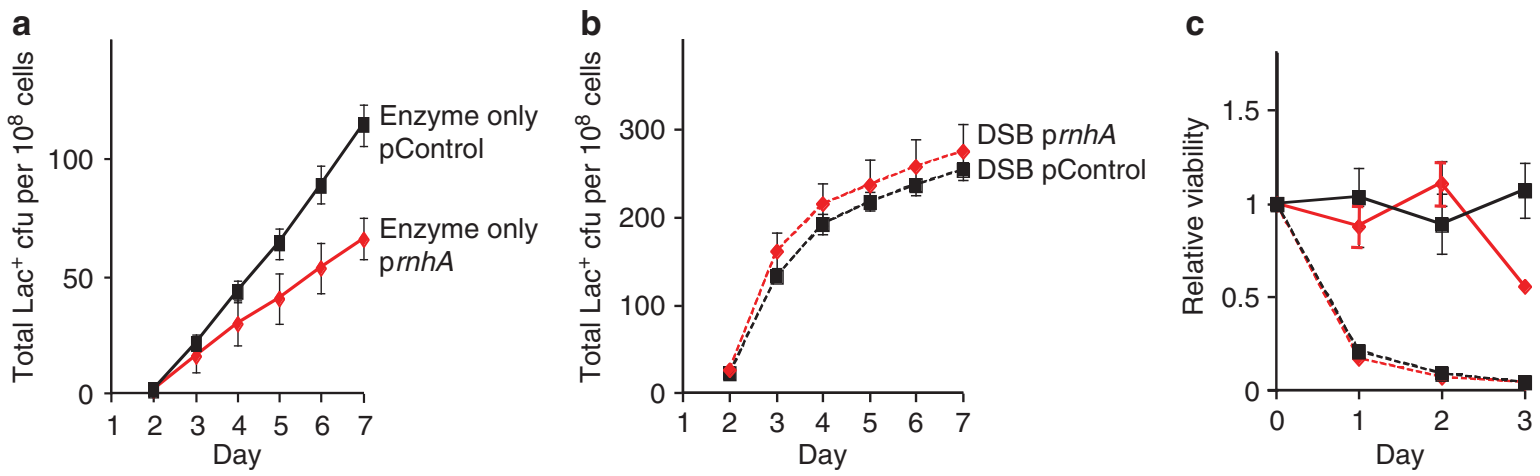

Figure 5 | R-loops are not needed for mutagenesis when DSBs are caused by I-Scel. Overproduction of RNase HI (reduction in R-loops) does not decrease mutagenesis when I-Scel-generated DSBs are provided. (a) The RNase HI-overproducing strain (PJH1239) ( $\bullet$ ) shows about half as much Lac + mutation as the strain carrying the control plasmid (PJH1237) ( $(\mathbf{a})$. (b) The DSB-producing strain (carrying the chromosomal inducible I-Scel enzyme and I-Scel cutsite near lac) that over-produces RNase HI (PJH1321) ( $\bullet$ shows no reduction of $\mathrm{Lac}^{+}$mutants compared with the isogenic strain carrying the control plasmid (PJH1319) ( $\mathbf{a})$, indicating that R-loops are not necessary for mutation when DSBs are provided. This result holds when both I-Scel enzyme and cutsite are present and not in strains with the enzyme only (a). (c) Strains experiencing double-strand cutting by I-Scel show reduced viability. However, the viability decrease is the same for cells with the rnhA plasmid as for those with the control plasmid making quantitative comparisons meaningful. Relative viability was determined per $^{21}$. Values in $(\mathbf{a}, \mathbf{b})$ have not been corrected for declining viability. Error bars represent one s.e.m. of four parallel cultures. Each experiment was performed three times with comparable results.

from its effect without I-SceI $(P=0.013$, Student's $t$-test). We conclude that R-loops contribute to DSB or DSE generation and so are not needed if DSBs are provided.

We could not distinguish amplification from point mutation in these experiments because of the low proportion of lac-amplified colonies. lac-amplified colonies appear later than point mutants ${ }^{36}$, and so are more affected by loss of viability over time. Also, although amplification is stimulated by I-SceI cuts made near lac, point mutation is stimulated more than amplification $^{21}$, because co-amplification of the cutsite with lac causes loss of the repeats ${ }^{21}$.

These results indicate that R-loops function in the mutagenesis pathway upstream of (leading to the production of) the DSBs or DSEs that initiate mutagenesis.

Formation of R-loop-promoted DSEs requires a ssDNA nick. In this section and the next, we provide two additional, independent lines of support for the conclusion that R-loops promote SIM by creation of DSEs. We also show that they do so in a twostep process that additionally requires a ssDNA nick.

First, we produced the DSE-binding Gam protein from phage $\mathrm{Mu}$ in RNase HI-deletion cells. Gam binds to DSEs and prevents them from being processed by nucleases ${ }^{45}$, and thus prevents DSE-induced recombination ${ }^{46}$. We produced Gam from the chromosome controlled by a doxycycline-inducible promoter. Doxycycline induction of Gam reduces amplification and point mutation (Fig. 6), confirming that both mechanisms require a DSE that is blocked by Gam. Importantly, induction of Gam prevents the increase of amplification and point mutation seen otherwise in RNase HI-defective cells, in which R-loops accumulate. These results show that the SIM promoted by R-loops in RNase HI-defective cells requires DSEs, and does not result from a different, DSE-independent pathway.

Second, we suggest a two-step model in which R-loops generate DSEs in stationary cells by priming replication forks that become DSEs when they encounter a ssDNA nick (Fig. 1a-f, Introduction). R-loops can prime replication independently of standard origins in RNase $\mathrm{H}$-defective cells ${ }^{34,35}$ and in solution with purified proteins ${ }^{19}$. In the $\mathrm{F}^{\prime}$ plasmid, ssDNA nicks are made constitutively by TraI, a site-specific ssDNA endonuclease that nicks the $\mathrm{F}^{\prime}$ transfer origin, and which is required for SIM at sites in the $\mathrm{F}^{21}$. The antimutagenic effect of deleting traI is more than suppressed by providing I-SceI-generated DSEs near $l a c^{21}$, showing that TraI contributes to formation of DSEs that drive mutagenesis, presumably by fork collapse at the ssDNA nick (Fig. 1e,f $)^{7,21}$. We suggest that R-loops cause DSEs by priming replication forks that collapse at a ssDNA nick (Fig. 1a-f). This model predicts that ssDNA nicks made by TraI will be required for the R-loop-mediated production of DSEs. Conversely, if R-loops promoted DSE formation independently of ssDNA nicks, we might find that although TraI is required for most mutagenesis in $\mathrm{RNase} \mathrm{HI}^{+}$cells $^{21}$, it would not be required for the extra mutagenesis observed in RNase HI-defective cells. Supporting the two-step R-loop-plus-ssDNA-nick model (Fig. 1), Fig. 7 shows that most mutation induced in RNase HI knockout cells requires TraI. Whereas $\Delta$ traI reduced mutation rate in otherwise wild-type cells about 60 -fold, it reduced the rate in RNase HI-defective cells 108-fold (Fig. 7, Supplementary Table S1), showing a very strong requirement for TraI in the mutagenesis promoted by R-loops. Thus, most DSEs generated by $\mathrm{R}$-loops in the $\mathrm{F}^{\prime}$ form in a process that also requires the ssDNA nick made by TraI.

A small fraction of the extra R-loop-promoted SIM in RNase HI null cells is TraI-independent, in that knock out of RNase HI increased mutation slightly but just significantly in TraI-defective cells (1.8-fold, mean of three experiments, $P=0.0376$, Student's $t$-test). This could result from spontaneous ssDNA nicks (Fig. 1a-f). We conclude that ssDNA nicks are required for most of the mutagenesis promoted by R-loops. This supports the hypothesis that R-loops generate the breaks in these stationary cells via the two-step mechanism in which the RNA primes replication that collapses at a ssDNA nick producing a DSE (Fig. 1a-f). This is unlike DSEs generated by co-directional collisions in replicating cells, in which the R-loop itself is thought to create a ssDNA nick ${ }^{18}$. In molecules other than F, spontaneous ssDNA nicks, which are ubiquitous and common ${ }^{47}$, are expected to play this role.

R-loops plus single-stranded nicks generate visible DSEs. We used a visual assay to show directly that R-loops generate DSEs, and that DSE formation requires both the R-loop and a ssDNA nick. We assayed DSEs in living cells by quantifying cells 

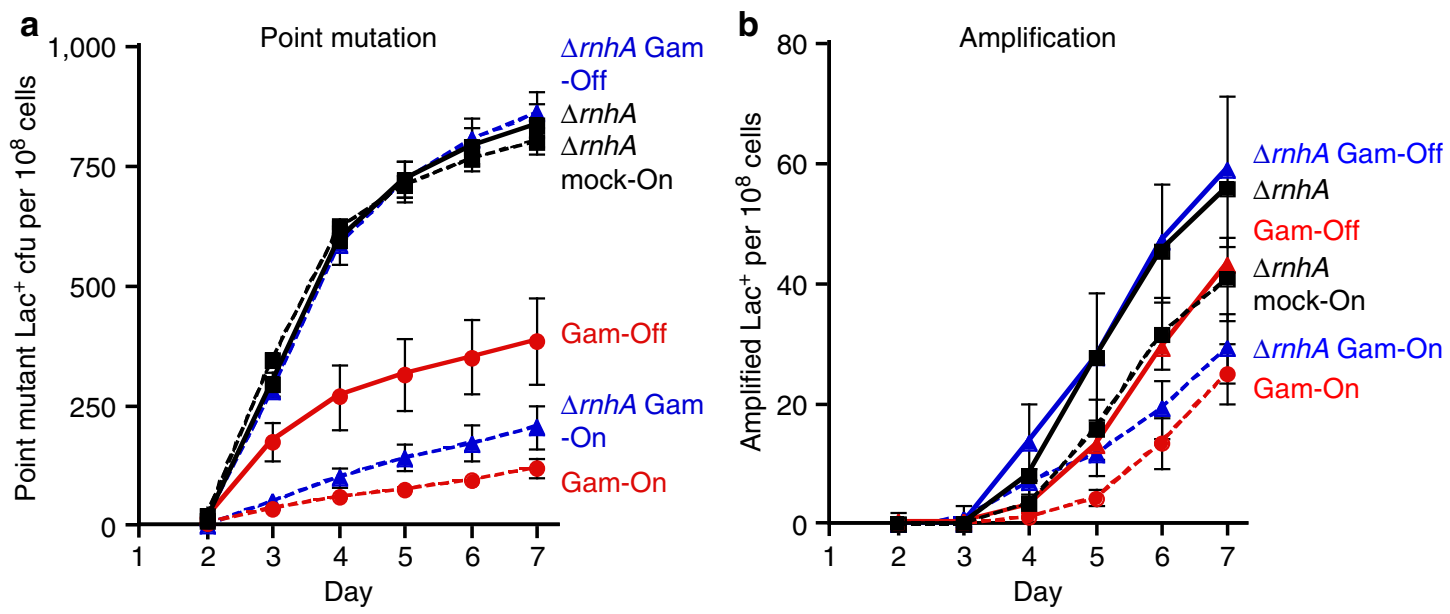

Figure 6 | Gam prevents R-loops from initiating amplification and point mutation. The increased amplification and mutation seen in cells with increased R-loops due to knock out of RNase $\mathrm{HI}(\Delta \mathrm{rnh} A)$ is prevented by doxycycline-induced production of Gam protein (Gam-On) (Methods). Gam binds DSEs ${ }^{45}$, prevents them engaging in repair ${ }^{46}$ and reduces (a) point mutation and (b) amplification, confirming that DSEs are required for both pathways. Doxycycline-induced production of Gam protein (Gam-On) prevents the increase in a point mutation and $\mathbf{b}$ amplification caused by knock out of RNase HI $(\Delta r n h A)$, demonstrating that the increased R-loops cause increased amplification and point mutation via DSEs. Strains: $\Delta r n h A$ tetR PJH2039 ( $\mathbf{\square}) ; \Delta r n h A$

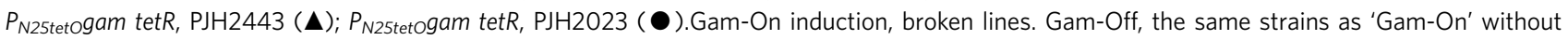
doxycycline induction; mock-On, strains with the Tet repressor but without the gam gene treated with doxycycline. Error bars represent one s.e.m. of four parallel cultures. This experiment was performed three times with comparable results.

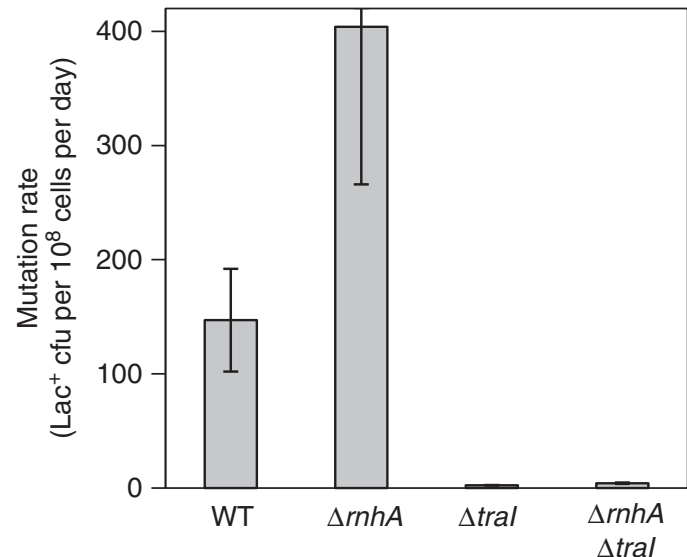

Figure 7 | R-loop-promoted mutagenesis requires SsDNA endonuclease

Tral. The abundance of R-loops caused by deletion of $r n h A$ is not sufficient, but also requires Tral-generated ssDNA nicks, to promote mutagenesis. Therefore generation of DSEs by R-loops requires both an R-loop and a ssDNA nick. Strains: WT, SMR4562; $\Delta r n h A$, PJH683; $\Delta$ tral, PJH234; $\Delta r n h A$ $\Delta$ tral, PJH 963. Mean \pm s.e.m. of three experiments.

with visible foci of the DSE-labelling GamGFP fusion protein (Methods) in the E. coli chromosome, and in the $\mathrm{F}^{\prime}$ plasmid in which amplification and point mutation were assayed. Figure $8 \mathrm{~b}$ shows a representative example of the results, which are quantified in Fig. 8a and Supplementary Table S2. The following points are evident (Supplementary Table S2, Fig. 8a): first, $24 \%$ of cells with an $\mathrm{F}^{\prime}$ showed foci, whereas only $6.3 \%$ of $\mathrm{F}^{-}$cells did, indicating that the difference (17.7\%) is foci caused by DSEs in the $\mathrm{F}^{\prime}$. Second, those $\mathrm{F}^{\prime}$-specific DSEs are mostly TraI-dependent, because they are reduced to $8 \%$ when TraI is deleted (the $6 \%$ in $\mathrm{F}^{-}$cells and $8 \%$ in cells with a TraI-defective $\mathrm{F}^{\prime}$ are not significantly different, $P=0.904$, Student's $t$-test). Third, knockout of RNase $\mathrm{HI}$ increased the number of $\mathrm{F}^{-}$and $\mathrm{F}^{\prime}$-carrying cells with foci to $38 \%$ (from $6.3 \%$ in WT) and $73 \%$ (from $24 \%$ in
$\left[\mathrm{F}^{\prime}\right]$ ), respectively, showing that R-loops promote DSE formation robustly in both the chromosome and $\mathrm{F}^{\prime}$. Fourth, most GamGFP foci in RNase HI-defective cells with an $\mathrm{F}^{\prime}$ are TraI-dependent. Whereas $73 \pm 2 \%$ of $\mathrm{F}^{\prime}$ RNase HI-deletion cells show foci, only $50 \pm 2 \%$ of $\mathrm{F}^{\prime}$ RNase HI-deletion cells lacking TraI do. This number is not significantly higher than the $38 \pm 4 \%$ of $\mathrm{F}^{-}$RNase HI-deletion cells that show foci (Supplementary Table S2, Fig. 8a, $P=0.07$, Student's $t$-test), suggesting that most of the increased DSBs caused by $\mathrm{F}$ are caused by TraI activity.

Overall, the data confirm that first, DSEs, assayed directly as GamGFP foci, are promoted by R-loops, and second, that the R-loop-promoted DSE foci also require a second event, a ssDNA nick, which in the $\mathrm{F}^{\prime}$ is usually generated by TraI. This supports the two-step R-loop-plus-nick model in Fig. 1a-f, in which $\mathrm{R}$-loops promote DSE formation by priming replication forks that collapse at a ssDNA nick.

\section{Discussion}

The results presented demonstrate a mechanism of generation of DSBs/DSEs by R-loops in starving, stationary-phase E. coli cells that requires Mfd translocase and also depends on ssDNA nicks. Because ssDNA nicks are common, spontaneously ${ }^{47}$ this mechanism is likely to be generally important. We suggest that replication forks initiated from R-loops in stationary cells collapse, generating DSEs at independently occurring ssDNA nicks (Fig. 1a-f). This model can explain how DSEs can occur in non-replicating cells, in which standard origin-dependent replication is inhibited, in contrast with most current models for spontaneous DSE production (reviewed in the Introduction).

R-loops were inferred previously to initiate replication in RNase HI- or RecG-defective cells ${ }^{35}$, and origin-independent replication (not demonstrably primed by R-loops) was seen in RNase $\mathrm{HI}^{+} \mathrm{RecG}^{+}$cells returned to growth upon entry to stationary phase ${ }^{48}$. This mechanism might be R-loopindependent and also does not resemble R-loop-promoted DNA breakage demonstrated here, in that it is RecB (DSB)independent ${ }^{48}$. 

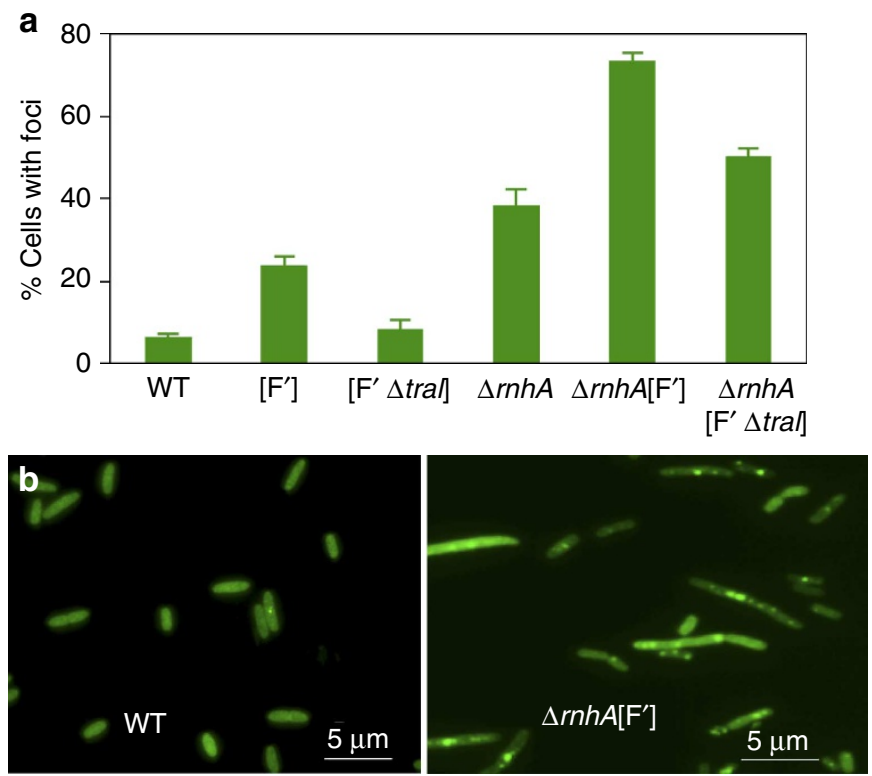

Figure 8 | Formation of DSEs promoted by R-loops also requires ssDNA nicks. (a) Quantification of cells with GamGFP foci. Cells carrying the $F^{\prime}$ plasmid show more foci than $\mathrm{F}^{-}$(WT) cells, and these extra foci are Traldependent, and thus result from the ssDNA nicks made by Tral endonuclease at oriT. Cells lacking RNase HI show more foci than their isogenic RNase $\mathrm{HI}^{+}$parents (compare WT with $\Delta r n h A$; and $\left[\mathrm{F}^{\prime}\right]$ with $\Delta r n h A\left[F^{\prime}\right]$ ) indicating that lack of RNase $\mathrm{HI}$ (increased R-loops) causes increased DSEs. Finally, the increased foci caused by loss of RNase $\mathrm{HI}$ in the $\mathrm{F}^{\prime}$ (compared with that in $\mathrm{F}^{-} \Delta r n h A$ cells) requires Tral ssDNA

endonuclease. Therefore, R-loop-mediated foci require both the R-loop and a ssDNA nick. Means \pm s.e.m. of three experiments. WT 'wild-type'

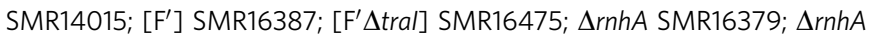
[F'] SMR16389; $\Delta r n h A\left[F^{\prime} \Delta\right.$ tral] SMR16477. All strains carry a chromosomal tet $R$ and $P_{\text {N25tetogam-gfp. Supplementary Table S2 for }}$ numerical values. (b) Examples of fields of cells showing few GamGFP foci in wild-type, SMR14015, and multiple foci in most cells in $\Delta r n h A\left[F^{\prime}\right]$, SMR16389.

Previous reports that cells lacking RNase HI and DSE-repair enzyme RecBCD are inviable suggested that RNase $\mathrm{H}$-defective cells might have more DSEs than wild-type ${ }^{35,49}$. The results presented here support this interpretation with more direct data (for example, Figs 5 and 8) and add to it that the R-loops are transcriptional (Figs 1-4) and that DSE generation by R-loops additionally requires a ssDNA nick (Figs 7 and 8 ), thus providing strong support for the specific mechanism shown in Fig. 1a-f.

R-loops are identified here as the earliest known molecular intermediate in stress-induced amplification and point mutation in E. coli. R-loops might also underlie other instances of DSE formation that instigates genomic instability in stressed cells in which replication is likely to be inhibited. For example, collapse of R-loop-generated forks might underlie the DSEs that drive bileinduced resistance mutagenesis in pathogenic Salmonella ${ }^{6}$, DSEs that create genetic diversity in biofilms of pathogenic Pseudomonas ${ }^{4}$, genomic instability leading to cancer initiation in cells of non-proliferating tissues, and cancer and pathogen resistance to growth-arresting chemotherapies. DSBs/DSEs of unknown origin are hypothesized to underlie mutation hotspots in cancer genomes ${ }^{50,51}$, as demonstrated in E. coli ${ }^{25}$, and might similarly be instigated by the R-loop-generated fork-collapse-atnick mechanism shown here.

$\mathrm{R}$-loops are also implicated in replication initiation in ColE1 plasmids ${ }^{52}$, mouse mitochondrial replication ${ }^{53}$ and class-switch recombination and somatic hypermutation in the mammalian immune system (reviewed by Chaudhuri and $\mathrm{Alt}^{54}$ ). R-loops are associated with hyper-recombination, genomic instability and DSBs from bacteria to yeast and human (reviewed by Li and Manley ${ }^{55}$ ). R-loops underlie breakage at common fragile sites in human $^{56}$. Some of these instances of breakage require replication as well as transcription ${ }^{18,56}$, and so might also result from R-loopgenerated fork collapse at nicks.

Transcription was implicated previously in DSB-dependent mutagenesis in $E$. coli in that a strain with a partially-defective NusA transcription anti-termination factor shows reduced SIM, and might do so via reduced interactions with DinB error-prone DNA polymerase ${ }^{57}$. Our data suggest a possible alternative explanation that could unite their data and ours. R-loops form preferentially at particular DNA sequences, hypothesized to have special structural features ${ }^{35}$. When NusA is functional, some transcripts will be longer (anti-terminated). Perhaps, in the presence of functional NusA, a transcript that would otherwise not reach a site prone to stable R-loop formation reaches such a site, such that R-loops are formed or stabilized, promoting SIM. This model and others remain to be tested.

The association of transcriptional R-loops with DSEs opens the possibility that in addition to being targeted preferentially to times of stress, by their coupling to the stress responses ${ }^{7,9}$ and see Al Mamun et $a l^{30}$ ), stress-induced point mutation and amplification could be targeted preferentially to regions of active transcription. DSE-dependent point mutagenesis occurs in strong hotpots maximally within two and up to $60 \mathrm{~kb}$ from a $\mathrm{DSE}^{25}$. This means that transcription-produced DSEs would be expected to cause hotspots of mutations near sites of transcription. The suggestion that DSBs cause the hotspots observed in cancer genomes and chemically damaged yeast cells ${ }^{50,51}$ suggests the possibility of transcriptional/R-loop targeting of mutations also in cancer genomes. Transcription also promotes local SIM in starving B. subtilis cells, though apparently independently of DSEs and recombination ${ }^{58}$.

In stressed cells, actively transcribed regions are likely to harbour genes whose products counter the stress. Thus, stressinduced point mutations and genome rearrangements might occur preferentially where they are most likely to affect phenotypes immediately, including advantageously. Both regulation of mutagenesis in time, by stress responses, and in genomic space, by linkage with transcription and DSEs, could enhance the ability to evolve during stress for small populations in which mutation supply is limiting.

\section{Methods}

Strains. Strains and plasmids used in this study are listed in Supplementary Table S3 and are isogenic with SMR4562, an independent construction of FC40 (ref. 29). FC40/SMR4562 carry a +1 frameshift mutation in a lacI-lacZ fusion gene in $\mathrm{F}^{\prime}$ plasmid $\mathrm{F}^{\prime} 128$. FC29 (ref. 29) is an $\mathrm{F}^{\prime}$-carrying non-revertible lac deletion strain that is used to scavenge extraneous carbon sources from minimal lactose plates. SMR5383, an ampicillin-resistant $\mathrm{ara}^{-}$scavenger derived from FC29, was used in experiments involving pBAD constructs or plasmids. $\Delta m f d$ and $\Delta r n h A$ alleles were introduced into strains by $\mathrm{P} 1$ transduction.

SIM experiments. $\mathrm{Lac}^{-}$E. coli were grown in minimal glycerol medium to prolonged stationary phase, plated onto minimal lactose solid medium and incubated for 7 days at $37^{\circ} \mathrm{C}^{22}$. The $\mathrm{Lac}^{+}$colonies that arise each day are counted and marked. They have acquired either a compensatory-frameshift ('point') mutation, or amplification of the leaky lac allele to 20 or more copies, which allows growth without a reversion mutation ${ }^{36}$. Amplification of lac is unstable under nonselective conditions, so lac-amplified clones can be identified by plating cells from $\mathrm{Lac}^{+}$ colonies onto nonselective medium containing 5-bromo-4-chloro-3-indoyl- $\beta$-Dgalactoside (X-gal) on which lac-amplified isolates show a sectored appearance. In spectinomycin-pulse experiments, $100 \mathrm{~g} \mathrm{ml}^{-1}$ spectinomycin was added $3 \mathrm{~h}$ before plating and then washed from cells before plating on minimal lactose medium. All experiments were performed $\geq 3$ times, and repeat experiments gave consistent results. Representative graphs were chosen for illustration and 
summaries of mutation rate data from multiple experiments (mutations per cell per day) are given in the text.

In experiments with plasmid pBAD18-rnhA overproducing RNase HI, $100 \mu \mathrm{g} \mathrm{ml}^{-1}$ ampicillin was added to the minimal lactose plates to maintain the plasmid. The arabinose is not catabolized (the cells still starve) because the cells are $\mathrm{ara}^{-}$. Plugs of agar from these plates were taken with capillary pipettes periodically during the experiment, resuspended and plated for colony-forming units on plates with and without ampicillin to verify that cells retained the plasmid. No significant plasmid loss was observed. To produce RNase HI, $5 \times 10^{-8}(\mathrm{w} / \mathrm{v})$ arabinose was added to the minimal lactose plates. This concentration was chosen because we found that higher concentrations caused inhibition of growth. The same concentrations of ampicillin and arabinose were used in experiments with I-SceI and overproduction of RNase HI. The arabinose induces production of both RNase HI and I-SceI. In I-SceI experiments, the number of ampicillin-resistant cells carrying the plasmid was similar for the control and RNase HI-producing plasmids $(0.77 \pm 0.09$ versus $0.70 \pm 0.16$, respectively, in cells recovered from lactose plates after 1 day, mean \pm s.e.m.), and did not change significantly during the experiment. The proportion of cells showing resistance to arabinose (indicating loss of the I-SceI cut site ${ }^{21}$ ) was not different between the DSB-inducing strains with and without the RNase HI plasmid $(0.19 \pm 0.02$ versus $0.22 \pm 0.02$, respectively, in the experiment shown)

For experiments in Fig. 6, Gam was induced by doxycycline at $100 \mathrm{ng} \mathrm{ml}^{-1}$ applied for $48 \mathrm{~h}$ from early stationary phase.

Inducible Gam and GAMGFP of phage Mu. Details of construction and validation of the chromosomally expressed inducible Gam and GamGFP will be published separately. In brief, the EcoRI fragment of the bacteriophage Mu gam gene from plasmid pJA2 $1^{59}$ was sub-cloned downstream from the $\mathrm{P}_{\mathrm{N} 25}$ tetO promoter ${ }^{60}$, a promoter that can be induced by tetracycline or doxycycline, in cells carrying $\mathrm{P}_{\mathrm{N} 25}$ tetR, encoding a constitutive Tet repressor, which represses transcription from $\mathrm{P}_{\mathrm{N} 25}$ tetO in non-inducing conditions ${ }^{60} . \mathrm{P}_{\mathrm{N} 25}$ tetOgam was placed in the E. coli chromosome. Similarly, the gam-gfp fusion gene, to be described elsewhere, was placed downstream of the $\mathrm{P}_{\mathrm{N} 25}$ tetO

For experiments in Fig. 8, production of GamGFP was induced in log-phase with $100 \mathrm{ng} \mathrm{ml}^{-1}$ doxycycline for $3 \mathrm{~h}$. Cells were concentrated and placed on slides and photographed in bright field and phase contrast. At least ten fields of $\sim 10-100$ cells were counted for each sample. Samples were scored blindly as focuscontaining or not.

\section{References}

1. Cousineau, I., Abaji, C. \& Belmaaza, A. BRCA1 regulates RAD51 function in response to DNA damage and suppresses spontaneous sister chromatid replication slippage: implications for sister chromatid cohesion, genome stability and carcinogenesis. Cancer Res. 65, 11384-11391 (2005).

2. Elliot, B. \& Jasin, M. Double-strand breaks and translocations in cancer. Cell. Mol. Life. Sci. 59, 373-385 (2002).

3. Debacker, K. \& Kooy, R. F. Fragile sites and human disease. Hum. Mol. Genet. 16 Spec no. 2 R150-R158 (2007).

4. Boles, B. R. \& Singh, P. K. Endogenous oxidative stress produces diversity and adaptability in biofilm communities. Proc. Natl Acad. Sci. USA 105, 12503-12508 (2008)

5. Cirz, R. T., Chin, J. K., Andes, D. R., de Crecy-Lagard, V., Craig, W. A. \& Romesberg, F. E. Inhibition of mutation and combating the evolution of antibiotic resistance. PLoS Biol. 3, el76 (2005).

6. Prieto, A. I., Ramos-Morales, F. \& Casadesus, J. Repair of DNA damage induced by bile salts in Salmonella enterica. Genetics 174, 575-584 (2006).

7. Galhardo, R. S., Hastings, P. J. \& Rosenberg, S. M. Mutation as a stress response and the regulation of evolvability. Crit. Rev. Biochem. Mol. Biol. 42, 399-435 (2007).

8. Hastings, P. J. Adaptive amplification. Crit. Rev. Biochem. Mol. Biol. 42, 1-13 (2007).

9. Rosenberg, S. M., Shee, C., Frisch, R. L. \& Hastings, P. J. Stress-induced mutation via DNA breaks in Escherichia coli: a molecular mechanism with implications for evolution and medicine. Bioessays 34, 885-892 (2012).

10. Vilenchik, M. M. \& Knudson, A. G. Endogenous DNA double-strand breaks: production, fidelity of repair, and induction of cancer. Proc. Natl Acad. Sci. USA 100, 12871-12876 (2003).

11. Pennington, J. M. \& Rosenberg, S. M. Spontaneous DNA breakage in single living cells of Escherichia coli. Nat. Genet. 39, 797-802 (2007).

12. Kuzminov, A. Single-strand interruptions in replicating chromosomes cause double-strand breaks. Proc. Natl Acad. Sci. USA 98, 8241-8246 (2001).

13. Seigneur, M., Bidnenko, V., Ehrlich, S. D. \& Michel, B. RuvAB acts at arrested replication forks. Cell 95, 419-430 (1998).

14. Kang, M. J., Lee, C. H., Kang, Y. H., Cho, I. T., Nguyen, T. A. \& Seo, Y. S. Genetic and functional interactions between Mus81-Mms4 and Rad27. Nucleic Acids Res. 38, 7611-7625 (2010).

15. Tehranchi, A. K. et al. The transcription factor DksA prevents conflicts between DNA replication and transcription machinery. Cell 141, 595-605 (2010).
16. Bermejo, R., Lai, M. S. \& Foiani, M. Preventing replication stress to maintain genome stability: resolving conflicts between replication and transcription. Mol. Cell. 45, 710-718 (2012).

17. Merrikh, H., Zhang, Y., Grossman, A. D. \& Wang, J. D. Replicationtranscription conflicts in bacteria. Nat. Rev. Microbiol. 10, 449-458 (2012).

18. Dutta, D., Shatalin, K., Epshtein, V., Gottesman, M. E. \& Nudler, E. Linking RNA polymerase backtracking to genome instability in E. coli. Cell 146, 533-543 (2011)

19. Pomerantz, R. T. \& O'Donnell, M. The replisome uses mRNA as a primer after colliding with RNA polymerase. Nature 456, 762-766 (2008).

20. Harris, R. S., Longerich, S. \& Rosenberg, S. M. Recombination in adaptive mutation. Science 264, 258-260 (1994).

21. Ponder, R. G., Fonville, N. C. \& Rosenberg, S. M. A switch from high-fidelity to error-prone DNA double-strand break repair underlies stress-induced mutation. Mol. Cell 19, 791-804 (2005).

22. Slack, A., Thornton, P. C., Magner, D. B., Rosenberg, S. M. \& Hastings, P. J. On the mechanism of gene amplification induced under stress in Escherichia coli. PLoS. Genet. 2, e48 (2006).

23. Lombardo, M. -J., Aponyi, I. \& Rosenberg, S. M. General stress response regulator RpoS in adaptive mutation and amplification in Escherichia coli. Genetics 166, 669-680 (2004).

24. Shee, C., Gibson, J. L., Darrow, M. C., Gonzales, C. \& Rosenberg, S. M. Impact of a stress-inducible switch to mutagenic repair of DNAbreaks on mutation in E. coli. Proc. Natl Acad. Sci. USA 108, 13659-13664 (2011).

25. Shee, C., Gibson, J. L. \& Rosenberg, S. M. Two mechanisms produce mutation hotspots at DNA breaks in Escherichia coli. Cell Rep. 2, 714-721 (2012).

26. Hastings, P. J., Ira, G. \& Lupski, J. R. A microhomology-mediated breakinduced replication model for the origin of human copy number variation. PLoS. Genet. 5, e1000327 (2009).

27. Lin, D., Gibson, I. B., Moore, J. M., Thornton, P. C., Leal, S. M. \& Hastings, P. J. Global chromosomal structural instability in a subpopulation of starving Escherichia coli cells. PLoS. Genet. 7, e1002223 (2011).

28. Hastings, P. J., Lupski, J. R., Rosenberg, S. M. \& Ira, G. Mechanisms of change in gene copy number. Nat. Rev. Genet. 10, 551-564 (2009).

29. Cairns, J. \& Foster, P. L. Adaptive reversion of a frameshift mutation in Escherichia coli. Genetics 128, 695-701 (1991).

30. Al Mamun, A. A. et al. Identity and function of a large gene network underlying mutagenic repair of DNA breaks. Science 338, 1344-1348 (2012).

31. Rodriguez, C., Tompkin, J., Hazel, J. \& Foster, P. L. Induction of a DNA nickase in the presence of its target site stimulates adaptive mutation in Escherichia coli. J. Bacteriol. 184, 5599-5608 (2002).

32. Kuzminov, A. Collapse and repair of replication forks in Escherichia coli. Mol. Microbiol. 16, 373-384 (1995).

33. Ito, A., May, T., Kawata, K. \& Okabe, S. Significance of rpoS during maturation of Escherichia coli biofilms. Biotechnol. Bioeng. 99, 1462-1471 (2008).

34. Kogoma, T. A novel Escherichia coli mutant capable of DNA replication in the absence of protein synthesis. J. Mol. Biol. 121, 55-69 (1978).

35. Kogoma, T. Stable DNA replication: interplay between DNA replication, homologous recombination, and transcription. Microbiol. Mol. Biol. Rev. 61, 212-238 (1997).

36. Hastings, P. J., Bull, H. J., Klump, J. R. \& Rosenberg, S. M. Adaptive amplification: an inducible chromosomal instability mechanism. Cell 103, 723-731 (2000).

37. Berkower, I., Leis, J. \& Hurwitz, J. Isolation and characterization of an endonuclease from Escherichia coli specific for ribonucleic acid in ribonucleic acid-deoxyribonucleic acid hybrid structures. J. Biol. Chem. 248, 5914-5921 (1973).

38. Park, J. S., Marr, M. T. \& Roberts, J. W. E. coli Transcription repair coupling factor (Mfd protein) rescues arrested complexes by promoting forward translocation. Cell 109, 757-767 (2002).

39. Selby, C. P. \& Sancar, A. Molecular mechanism of transcription-repair coupling. Science 260, 53-58 (1993).

40. Pomerantz, R. T. \& O'Donnell, M. Direct restart of a replication fork stalled by a head-on RNA polymerase. Science 327, 590-592 (2010).

41. Masse, E. \& Drolet, M. Escherichia coli DNA topoisomerase I inhibits R-loop formation by relaxing transcription-induced negative supercoiling. J. Biol. Chem. 274, 16659-16664 (1999).

42. Bilgin, N., Richter, A. A., Ehrenberg, M., Dahlberg, A. E. \& Kurland, C. G. Ribosomal RNA and protein mutants resistant to spectinomycin. $E M B O ~ J .9$, 735-739 (1990).

43. McKenzie, G. J., Lee, P. L., Lombardo, M. -J., Hastings, P. J. \& Rosenberg, S. M. SOS mutator DNA polymerase IV functions in adaptive mutation and not adaptive amplification. Mol. Cell 7, 571-579 (2001).

44. Gibson, J. L. et al. The $\sigma \mathrm{E}$ stress response is required for stress-induced mutagenesis in Escherichia coli. Mol. Micro. 77, 415-430 (2010). 
45. Williams, J. G. \& Radding, C. M. Partial purification and properties of an exonuclease inhibitor induced by bacteriophage Mu-1. J. Virol. 39, 548-558 (1981).

46. Thaler, D. S., Stahl, M. M. \& Stahl, F. W. Evidence that the normal route of replication-allowed Red-mediated recombination involves double-chain ends. EMBO. J. 6, 3171-3176 (1987).

47. Caldecott, K. W. Single-strand break repair and genetic disease. Nat. Rev. Genet. 9, 619-631 (2008).

48. Hong, X., Cadwell, G. W. \& Kogoma, T. Activation of stable DNA replication in rapidly growing Escherichia coli at the time of entry to stationary phase. Mol. Microbiol. 21, 953-961 (1996).

49. Itaya, M. \& Crouch, R. J. A combination of RNase H (rnh) and recBCD or sbcB mutations in Escherichia coli K12 adversely affects growth. Mol. Gen. Genet. 227, 424-432 (1991).

50. Nik-Zainal, S. et al. Mutational processes molding the genomes of 21 breast cancers. Cell 149, 979-993 (2012).

51. Roberts, S. A. et al. Clustered mutations in yeast and in human cancers can arrise from damaged long single-strand DNA regions. Mol. Cell 46, 424-435 (2012).

52. Itoh, T. \& Tomizawa, J. Formation of an RNA primer for initiation of replication of ColE1 DNA by ribonuclease H. Proc. Natl Acad. Sci. USA 77, 2450-2454 (1980).

53. Xu, B. \& Clayton, D. A. A persistent RNA-DNA hybrid is formed during transcription at a phylogenetically conserved mitochondrial DNA sequence. Mol. Cell. Biol. 15, 580-589 (1995).

54. Chaudhuri, J. \& Alt, F. W. Class-switch recombination: interplay of transcription, DNA deamination and DNA repair. Nat. Rev. Immunol. 4, 541-552 (2004)

55. Li, X. \& Manley, J. L. Cotranscriptional processes and their influence on genome stability. Genes Dev. 20, 1838-1847 (2006).

56. Helmrich, A., Ballarino, M. \& Tora, L. Collision between replication and transcription complexes cause common fragile site instability at the longest human genes. Mol. Cell 44, 966-977 (2011).

57. Cohen, S. E. \& Walker, G. C. The transcription elongation factor NusA is required for stress-induced mutagenesis in Escerichia Coli. Curr. Biol. 20, 80-85 (2010).

58. Martin, H. A., Pedraza-Reyes, M., Yasbin, R. E. \& Robleto, E. A. Transcriptional de-repression and Mfd are mutagenic in stressed Bacillus subtilis cells. J. Mol. Microbiol. Biotechnol. 21, 45-58 (2011).
59. Akroyd, J. E., Clayson, E. \& Higgins, N. P. Purification of the gam gene-product of bacteriophage $\mathrm{Mu}$ and determination of the nucleotide sequence of the gam gene. Nucleic Acids Res. 14, 6901-6914 (1986).

60. Nishihara, K., Kanemori, M., Kitagawa, M., Yanagi, H. \& Yura, T. Chaperone coexpression plasmids: differential and synergistic roles of DnaK-DnaJ-GrpE and GroEL-GroES in assisting folding of an allergen of Japanese cedar pollen, Cryj2, in Escherichia coli. Appl. Environ. Microbiol. 64, 1694-1699 (1998).

\section{Acknowledgements}

We are grateful to Marc Drolet for plasmids and to David Bates, Ryan Frisch, Caleb Gonzales, Christophe Herman, Jessica Moore and Jade Wang for critical reading of the manuscript, and D Bates and C Herman for helpful discussions. Supported by National Institutes of Health Grants R01-GM53158 (SMR), DP-CA174424 (SMR) and R01GM64022 (PJH).

\section{Author contributions}

P.J.H. conceived the study. H.W., S.M.R. and P.J.H. conceived the experiments. P.J.H. directed the experiments. H.W., C.S., P.C.T. and P.S. constructed strains, performed experiments and analysed data. S.M.R. and P.J.H. also interpreted data. H.W., S.M.R and P.J.H. wrote the paper.

\section{Additional information}

Supplementary Information accompanies this paper at http://www.nature.com/ naturecommunications

Competing financial interests: The authors declare no competing financial interests.

Reprints and permission information is available online at http://npg.nature.com/ reprintsandpermissions/

How to cite this article: Wimberly, H. et al. R-loops and nicks initiate DNA breakage and genome instability in non-growing Escherichia coli. Nat. Commun. 4:2115 doi: $10.1038 /$ ncomms3115 (2013).

This work is licensed under a Creative Commons AttributionNonCommercial-NoDerivs 3.0 Unported License. To view a copy of his license, visit http://creativecommons.org/licenses/by-nc-nd/3.0/ 


\section{Corrigendum: R-loops and nicks initiate DNA breakage and genome instability in non-growing Escherichia coli}

Hallie Wimberly, Chandan Shee, P.C. Thornton, Priya Sivaramakrishnan, Susan M. Rosenberg

$\&$ P.J. Hastings

Nature Communications 4:2115 doi:10.1038/ncomms3115 (2013); Published 5 Jul 2013; Updated 20 Jan 2014

In Supplementary Table S3 of this Article, some of the genotype entries are incorrect, as follows:

For the SMR6280 strain, SMR4562 $\Delta$ araBAD567 [F’ mhpA32miniTn7Kan(I-SceI cutsite)] should be SMR4562 $\Delta a r a B A D 567$ $\Delta a t t B c:: \mathrm{P}_{\mathrm{BAD}} \mathrm{I}-\mathrm{Sce}$ I [F' $m h p A 32:: \operatorname{mini} T n 7 \operatorname{Kan}(\mathrm{I}-S c e$ I cutsite)].

For the PJH813 strain, SMR4562 $\Delta$ mfd::FRTKanFRT should be SMR4562 $\Delta m f d:$ FRT.

For the PJH2023 strain, SMR4562 $\Delta$ attTn7::PN25tetOgam-gfpFRT $\Delta$ zfd2509.2::PN25tetRFRT should be SMR4562 $\Delta a t t T n 7::$ $\mathrm{P}_{\text {N25tetogamFRTcatFRT } \Delta z f d 2509.2:: \mathrm{P}_{\text {N25 }} \text { tetRFRT. }}$

For the PJH2443 strain, SMR4562 $\Delta$ attTn7::PN25tetOgam-gfpFRT $\Delta$ rnhA::FRTKanFRT $\Delta$ zfd2509.2::PN25tetRFRT should be SMR4562 $\Delta a t t T n 7:: \mathrm{P}_{N 25 t e t O} g a m$ FRTcatFRT $\Delta r n h A::$ FRTKanFRT $\Delta z f d 2509.2:: \mathrm{P}_{N 25}$ tetRFRT. 